Georgian Mathematical Journal

Volume 12 (2005), Number 2, 369-376

\title{
ON THE ASYMPTOTIC BEHAVIOUR OF SOLUTIONS OF THIRD ORDER DELAY DIFFERENTIAL EQUATIONS
}

\author{
SESHADEV PADHI
}

\begin{abstract}
Sufficient conditions in terms of coefficient functions have been obtained so that all nonoscillatory solutions along with their first and second derivatives of the third order delay differential equation

$$
y^{\prime \prime \prime}(t)+a(t) y^{\prime \prime}(t)+b(t) y^{\prime}(t)+c(t) y(g(t))=0
$$

tend to zero as $t \rightarrow \infty$.

2000 Mathematics Subject Classification: 34C10, 34C11.

Key words and phrases: Oscillation, nonoscillation, disconjugate, asymptotic behaviour, delay differential equations.
\end{abstract}

1. This paper is concerned with the asymptotic behaviour of nonoscillatory solutions of third order equations of the form

$$
y^{\prime \prime \prime}(t)+a(t) y^{\prime \prime}(t)+b(t) y^{\prime}(t)+c(t) y(g(t))=0,
$$

where $a, b, c$ and $g \in C([\sigma, \infty), R), \sigma \in R, g(t) \leq t$ and $g(t) \rightarrow \infty$ as $t \rightarrow \infty$.

Recently in [8] and [9], the authors have obtained a relationship between the asymptotic behaviour of nonoscilatory solutions of (1.1) and the ordinary differential equation

$$
y^{\prime \prime \prime}(t)+a(t) y^{\prime \prime}(t)+b(t) y^{\prime}(t)+c(t) y(t)=0
$$

for two cases: (i) $a(t) \geq 0, b(t) \leq 0$ and $c(t)>0$; (ii) $a(t) \leq 0, b(t) \leq 0$ and $c(t)<0$. This has been performed by using the canonical transformation due to Trench [14] and some comparison theorems due to Kusano and Naito [6]. Thus it is possible to predict the behaviour of nonoscilatory solutions of (1.1) if we know solutions of (1.2). It seems that it is not easy to study the asymptotic behaviour of solutions of (1.1) directly. One can observe that the techniques employed in [8] and [9] cannot be applied to studying the behaviour of solutions of (1.1) when $a(t) \geq 0, b(t) \geq 0$ and $c(t)>0$. We have used a different technique to relate the asymptotic behaviour of nonoscillatory solutions of (1.1) to that of the oscillation of (1.2).

A solution $y(t)$ of (1.1) or (1.2) is said to be oscillatory if it has arbitrarily large zeros and nonoscillatory if there exists $T \geq \sigma$ such that $y(t)>0$ or $<0$ for $t \geq T$. As usual, equation (1.2) is called oscillatory if it admits an oscillatory solution and nonoscillatory otherwise. Equation (1.2) is said to be disconjugate 
if none of nontrivial solutions of (1.2) has more than two zeros, counting multiplicities. On the other hand, equation (1.1) is said to be oscillatory if all its solutions are oscillatory and nonoscillatory if it admits an oscillatory solution. In [11], the authors have obtained sufficient conditions under which equation (1.1) with $a(t) \equiv 0$ and $b(t) \equiv 0$ is oscillatory.

2. This section deals with the asymptotic behaviour of nonoscillatory solutions of (1.1). Throughout this section we assume that $a(t) \geq 0, b(t) \geq 0$ and $c(t)>0$ for $t \geq \sigma$. We assume that

$$
c(t) \geq d>0, \quad c(t)-a(t) b(t)-b^{\prime}(t) \geq 0 \text { and } b(t) \text { is bounded. }
$$

We can write (1.1) and (1.2) in the forms

$$
\left(r(t) y^{\prime \prime}(t)\right)^{\prime}+q(t) y^{\prime}(t)+p(t) y(g(t))=0
$$

and

$$
\left(r(t) y^{\prime \prime}(t)\right)^{\prime}+q(t) y^{\prime}(t)+p(t) y(t)=0
$$

where $r(t)=e^{\int_{\sigma}^{t} a(s) d s}, q(t)=r(t) b(t)$ and $p(t)=r(t) c(t)$.

The following theorem in [10] will be needed for our use in the sequel.

Theorem 2.1. Suppose that $2 c(t)-a(t) b(t)-b^{\prime}(t) \geq 0$ holds. If $a^{\prime}(t) \leq 0$ and $\int_{\sigma}^{\infty} c(t) d t=\infty$, then (1.2) is oscillatory.

Lemma 2.2. Let $2 c(t)-a(t) b(t)-b^{\prime}(t) \geq 0$ and (1.2) be oscillatory. Then a solution $y(t)$ of (1.2) is nonoscillatory if and only if $F[y(t)]<0$ for $t \geq \sigma$, where

$$
F[y(t)]=r(t)\left(y^{\prime}(t)\right)^{2}-2 r(t) y(t) y^{\prime \prime}(t)-q(t) y^{2}(t)
$$

This follows from Lemma 5 in [3].

Theorem 2.3. Let $(\mathbf{H})$ hold. If (1.2) is oscillatory, then every nonoscillatory solution $y(t)$ of (1.2) satisfies the property

$$
\lim _{t \rightarrow \infty} y(t)=\lim _{t \rightarrow \infty} y^{\prime}(t)=\lim _{t \rightarrow \infty} y^{\prime \prime}(t)=0 .
$$

Proof. Since $2 c(t)-a(t) b(t)-b^{\prime}(t) \geq 0$, from Lemma 2.2 it follows that $F[y(t)]<$ 0 for $t \geq \sigma$. If $y\left(t_{0}\right)=0$ for some $t_{0} \in[\sigma, \infty)$, we have $F\left[y\left(t_{0}\right)\right] \geq 0$, a contradiction. Hence $y(t) \neq 0$ for some $t \in[\sigma, \infty)$. Without any loss of generality we may assume that $y(t)>0$ for $t \geq \sigma$. As $r(t)>1$ and $F[y(t)]<0$ for $t \geq \sigma$; then

$$
0 \leq\left(y^{\prime}(t)\right)^{2} \leq r(t)\left(y^{\prime}(t)\right)^{2}<y(t)\left(2 r(t) y^{\prime \prime}(t)+q(t) y(t)\right)
$$

Thus

$$
2 r(t) y^{\prime \prime}(t)+q(t) y(t)>0, t \geq \sigma
$$

Consequently, from (2.2) and (2.5) we obtain

$$
\begin{aligned}
0 \leq r(t) & y^{\prime \prime}(t)+q(t) y(t) \\
= & r(\sigma) y^{\prime \prime}(\sigma)+q(\sigma) y(\sigma)+\int_{\sigma}^{t}\left(q^{\prime}(s)-p(s)\right) y(s) d s \leq k,
\end{aligned}
$$


where $k=r(\sigma) y^{\prime \prime}(\sigma)+r(\sigma) y(\sigma)$. Then

$$
2 r(t) y^{\prime \prime}(t)+q(t) y(t) \leq 2 k .
$$

Using (2.4) and (2.7), we get

$$
\left(y^{\prime}(t)\right)^{2} \leq 2 k y(t)
$$

Further, from (2.3), for $t \geq \sigma$

$$
\begin{aligned}
0>F[y(t)] & \geq F[y(\sigma)]+\int_{\sigma}^{t}\left(2 p(s)-q^{\prime}(s)\right) y^{2}(s) d s \\
& \geq F[y(\sigma)]+\int_{\sigma}^{t}\left(2 c(s)-a(s) b(s)-b^{\prime}(s)\right) y^{2}(s) d s \\
& \geq F[y(\sigma)]+\int_{\sigma}^{t} c(s) y^{2}(s) d s \\
& \geq F[y(\sigma)]+d \int_{\sigma}^{t} y^{2}(s) d s .
\end{aligned}
$$

This inequality implies that $y \in L^{2}([\sigma, \infty), R)$. Proceeding as in the proof of Theorem 3.6 in [7], one may show that $\lim _{t \rightarrow \infty} y(t)=0$. We consider two cases, viz.,

$$
\int_{\sigma}^{\infty} a(t) d t=\infty
$$

and

$$
\int_{\sigma}^{\infty} a(t) d t<\infty
$$

First, we suppose that (2.9) holds. From (2.6) we obtain

$$
k \geq \int_{\sigma}^{t}\left(p(s)-q^{\prime}(s)\right) y(s) d s=\int_{\sigma}^{t}\left(c(s)-a(s) b(s)-b^{\prime}(s)\right) r(s) y(s) d s .
$$

Hence

$$
\int_{\sigma}^{\infty}\left(c(s)-a(s) b(s)-b^{\prime}(s)\right) r(s) y(s) d s \leq k
$$

Since

$$
\begin{aligned}
{\left[r(t) y^{\prime \prime}(t)+q(t) y(t)\right]^{\prime} } & =-\left(p(t)-q^{\prime}(t)\right) y(t) \\
& =-\left(c(t)-a(t) b(t)-b^{\prime}(t)\right) r(t) y(t) \\
& \leq 0
\end{aligned}
$$


by (2.6) we get

$$
\lim _{t \rightarrow \infty}\left[r(t) y^{\prime \prime}(t)+q(t) y(t)\right]=\ell, 0 \leq \ell<\infty .
$$

Since equations (1.2) and (2.2) are equivalent, by integrating (2.2) from $t$ to $s(\sigma<t<s)$ and then taking the limit as $t \rightarrow \infty$ we have

$$
r(t) y^{\prime \prime}(t)+q(t) y(t)=\ell+\int_{t}^{\infty}\left(p(s)-q^{\prime}(s)\right) y(s) d s
$$

which by virtue of (2.11) gives

$$
\begin{aligned}
y^{\prime \prime}(t)+b(t) y(t) & \leq\left[\ell+\int_{t}^{\infty}\left(c(s)-a(s) b(s)-b^{\prime}(s)\right) r(s) y(s) d s\right] r^{-1}(t) \\
& \leq(\ell+k) r^{-1}(t) .
\end{aligned}
$$

This inequality in turn implies $\lim _{t \rightarrow \infty}\left[y^{\prime \prime}(t)+b(t) y(t)\right]=0$. Since $b(t)$ is bounded and $\lim _{t \rightarrow \infty} y(t)=0$, it follows that $\lim _{t \rightarrow \infty} y^{\prime \prime}(t)=0$. Next suppose that $(2.10)$ holds. Clearly, (2.12) holds too. Since $b(t)$ is bounded, we have $\lim _{t \rightarrow \infty} r(t) y^{\prime \prime}(t)=$ $\alpha, 0 \leq \alpha<\infty$. Clearly, (2.10) implies $\int_{\sigma}^{\infty} \frac{1}{r(t)} d t=\infty$ and hence $\alpha>0$ yields $y^{\prime}(t) \rightarrow \infty$ as $t \rightarrow \infty$, a contradiction. Hence $\alpha=0$. Consequently, $\lim _{t \rightarrow \infty} y^{\prime \prime}(t)=0$. This completes the proof of the theorem.

Remark 1 . The boundedness of $b(t)$ is not needed when (2.9) holds in Theorem 2.3 .

Remark 2. Theorem 2.3 partially generalizes Theorem 24 in [4].

Corollary 2.4. Suppose that the conditions of Theorem 2.3 are satisfied. If $a^{\prime}(t) \leq 0$, then every nonoscillatory solution of (1.2) satisfies the property

$$
\lim _{t \rightarrow \infty} y(t)=\lim _{t \rightarrow \infty} y^{\prime}(t)=\lim _{t \rightarrow \infty} y^{\prime \prime}(t)=0 .
$$

This follows from Theorems 2.1 and 2.3.

The objective of this section is to obtain a result similar to Corollary 2.4 for the delay differential equation (1.1). We begin with the following lemma.

Lemma 2.5. Suppose that the second order differential equation

$$
z^{\prime \prime}+a(t) z^{\prime}+b(t) z=0
$$

is nonoscillatory. If $y(t)$ is a nonoscillatory solution of (1.1), then there exists $t_{0} \in[\sigma, \infty)$ such that $y(t) y^{\prime}(t)>0$ or $y(t) y^{\prime}(t)<0$ for $t \leq t_{0}$.

Proof. Suppose that $y(t)$ is a nonoscillatory solution of (1.1). Then there exists $t_{1} \geq \sigma$ such that $y(t)>0$ or $y(t)<0$ for $t \geq t_{1}$. Let $t_{2}>t_{1}$ be such that $g(t)>t_{1}$ for $t \geq t_{2}$. Hence $y(g(t))>0$ or $<0$ for $t \geq t_{2}$. Clearly, $-y^{\prime}(t)$ is a solution of the second order nonhomogeneous equation

$$
\left(r(t) z^{\prime}\right)^{\prime}+q(t) z=-p(t) y(g(t)), \quad t \geq t_{2}
$$


Since (2.13) is nonoscillatory, from the result due to Keener [5] it follows that all solutions of (2.14) are nonoscillatory. Hence, in particular, $y^{\prime}(t)$ is nonoscillatory. Consequently, there exists $t_{0} \geq t_{2}$ such that $y(t) y^{\prime}(t)>0$ or $y(t) y^{\prime}(t)<0$ for $t \leq t_{0}$. Thus the lemma is proved.

Theorem 2.6. Let $(\mathbf{H})$ and (2.10) hold and (2.13) be nonoscillatory. If for any $\mu \in\left(0, \frac{1}{2}\right)$, the third order differential equation

$$
u^{\prime \prime \prime}+a(t) u^{\prime \prime}+b(t) u^{\prime}+\mu\left(\frac{g(t)}{t}\right)^{2} c(t) u=0
$$

admits an oscillatory solution, then every nonoscillatory solution of (1.1) along with its first and second derivatives tends to zero as $t \rightarrow \infty$.

Proof. Let $y(t)$ be a nonoscillatory solution of (1.1). Without any loss of generality we may assume that $y(t)>0$ for $t \geq t_{0} \geq \sigma$. Thus there exists $t_{1} \geq t_{0}$ such that $y(g(t))>0$ for $t \geq t_{1}$. By Lemma 2.5, there exists $t_{2} \geq t_{1}$ such that $y^{\prime}(t)>0$ or $<0$ for $t \geq t_{2}$. Suppose that $y^{\prime}(t)>0$ for $t \geq t_{2}$. Then by (2.1), $\left(r(t) y^{\prime \prime}(t)\right)^{\prime}<0$ for $t \geq t_{2}$ and hence $y^{\prime \prime}(t)>0$ or $<0$ for $t \geq t_{3} \geq t_{2}$. Clearly, (2.10) implies that $\int_{\sigma}^{\infty} \frac{1}{r(t)} d t=\infty$. If $y^{\prime \prime}(t)<0$ for $t \geq t_{3}$, then the repeated integration of $\left(r(t) y^{\prime \prime}(t)\right)^{\prime}<0$ from $t_{3}$ to $t$ yields $y^{\prime}(t)<0$ for large $t$, a contradiction. Hence $y^{\prime \prime}(t)>0$ for $t \geq t_{3}$. This in turn implies $y^{\prime \prime \prime}(t)<0$ for $t \geq t_{3}$. For every $\mu \in\left(0, \frac{1}{2}\right)$, there exists $T_{\mu}>t_{3}$ such that

$$
\frac{y(g(t))}{y(t)} \geq \mu\left(\frac{g(t)}{t}\right)^{2}
$$

for $t \geq T_{\mu}$ (see Theorem 2.2 in [2]). Setting $z(t)=y^{\prime}(t) / y(t)$ for $t \geq T_{\mu}$, we get $z^{\prime}(t)+z^{2}(t)=y^{\prime \prime}(t) / y(t)$. Further, taking $u(t)=e^{\int_{T_{\mu}}^{t} z(s) d s}$ and using (2.16) we obtain

$$
u^{\prime \prime \prime}+a(t) u^{\prime \prime}+b(t) u^{\prime}+\mu\left(\frac{g(t)}{t}\right)^{2} c(t) u \leq 0
$$

for $t \geq T_{\mu}$. From Lemma 4 in [3], it follows that (2.15) is disconjugate on $\left[T_{\mu}, \infty\right)$, a contradiction. Hence $y^{\prime}(t)<0$ for $t \geq t_{2}$. From (2.3) and equation (2.1) we obtain for $t \geq t_{2}$

$$
F^{\prime}[y(t)] \geq r^{\prime}(t)\left(y^{\prime}(t)\right)^{2}+\left(2 p(t)-q^{\prime}(t)\right) y^{2}(t)>0 .
$$

Hence $F[y(t)]<0$ or $>0$ for $t \geq t_{4} \geq t_{2}$. We claim that $F[y(t)]<0$ for $t \geq t_{4}$. Since $y^{\prime}(t)<0$ for $t \geq t_{2}$, there are three possibilities on $y^{\prime \prime}(t)$, i.e., there exists $t_{5} \geq t_{2}$ such that $y^{\prime \prime}(t)>0$ or $<0$ or $y^{\prime \prime}(t)$ changes the sign for $t \geq t_{5}$. Let $t_{6}=\max \left(t_{5}, t_{6}\right)$. Clearly, $y^{\prime \prime}(t)<0$ for $t \geq t_{6}$ implies that $y(t)<0$ for large $t$, a contradiction. If $y^{\prime \prime}(t)>0$ for $t \geq t_{6}$, then $\lim _{t \rightarrow \infty} y^{\prime}(t)$ exists and $\leq 0$. From (2.17) we obtain for $t \geq t_{6}$

$$
F[y(t)] \geq F\left[y\left(t_{6}\right)\right]+\int_{t_{6}}^{t}\left(2 c(s)-a(s) b(s)-b^{\prime}(s)\right) r(s) y^{2}(s) d s
$$


and hence

$$
r(t)\left(y^{\prime}(t)\right)^{2} \geq \int_{t_{6}}^{t}\left(2 c(s)-a(s) b(s)-b^{\prime}(s)\right) r(s) y^{2}(s) d s .
$$

Taking the limit as $t \rightarrow \infty$ in (2.19), we have

$$
\lim _{t \rightarrow \infty} r(t)\left(y^{\prime}(t)\right)^{2}=\lambda>0 \text {. }
$$

Hence $\lim _{t \rightarrow \infty} y^{\prime}(t)=\lambda_{1}, \lambda_{1}<0$. This in turn implies $y(t)<0$ for large $t$, a contradiction. If $y^{\prime \prime}(t)$ changes the sign for $t \geq t_{6}$, then $y^{\prime}(t)$ has maxima for arbitrarily large $t$. We claim that $\lim \sup y^{\prime}(t)=0$. If not, then $\lim \sup y^{\prime}(t)<0$. Then for $0<\epsilon<-k$ there exists $\stackrel{t \rightarrow \infty}{T} \geq t_{6}$ such that $y^{\prime}(t)<\stackrel{t \rightarrow \infty}{k+\epsilon}$ for $t \geq T$. This in turn implies that $y(t)<0$ for large $t$, a contradiction. Hence our claim holds, i.e., $\lim \sup y^{\prime}(t)=0$. Let $\left\{t_{n}\right\}$ be the sequence of maxima of $y^{\prime}(t)$. So $\limsup y^{\prime}\left(t_{n}\right)=0$. Clearly, $\left\{t_{n}\right\}$ contains a subsequence $\left\{s_{n}\right\}, s_{n} \rightarrow \infty$ as $n \rightarrow \infty$ and $\lim _{n \rightarrow \infty} y^{\prime}\left(s_{n}\right)=0$. We may note that $y^{\prime \prime}\left(s_{n}\right)=0, n=1,2,3, \ldots$. Hence from (2.19)

$$
0=\lim _{n \rightarrow \infty} r\left(s_{n}\right)\left(y^{\prime}\left(s_{n}\right)\right)^{2} \geq \lim _{n \rightarrow \infty} \int_{t_{5}}^{s_{n}}\left(2 c(s)-a(s) b(s)-b^{\prime}(s)\right) r(s) y^{2}(s) d s>0,
$$

a contradiction. Thus our claim holds, i.e., $F[y(t)]<0$ for $t \geq t_{4}$. Clearly, for $t \geq t_{4},(2.6),(2.7)$ and $(2.8)$ are satisfied. From (2.8) it follows that $y^{\prime}(t)$ is bounded. One may obtain from (2.18)

$$
\int_{t_{4}}^{t} y^{2}(s) d s \leq \frac{-F\left[y\left(t_{4}\right)\right]}{d}<\infty
$$

Hence

$$
\int_{t_{4}}^{t} y^{2}(s) d s<\infty
$$

Now Lemma 1.2 of Singh [12] implies $y(t) \rightarrow 0$ as $t \rightarrow \infty$. Thus from (2.8) it follows that $y^{\prime}(t) \rightarrow 0$ as $t \rightarrow \infty$. Since (1.1) and (2.1) are equivalent, from (2.1) we have

$$
\begin{aligned}
{\left[r(t) y^{\prime \prime}(t)+q(t) y(t)\right]^{\prime} } & \leq-\left(p(t)-q^{\prime}(t)\right) y(t) \\
& \leq-\left(c(t)-a(t) b(t)-b^{\prime}(t)\right) r(t) y(t) \leq 0
\end{aligned}
$$

for large $t$. Hence $\left(r(t) y^{\prime \prime}(t)+q(t) y(t)\right)>0$ or $<0$ for large $t$. Clearly, $\left(r(t) y^{\prime \prime}(t)+q(t) y(t)\right)<0$ for large $t$ implies that $y(t)<0$ for large $t$, a contradiction. Hence $\left(r(t) y^{\prime \prime}(t)+q(t) y(t)\right)>0$ for large $t$. Consequently, $\lim _{t \rightarrow \infty}\left[r(t) y^{\prime \prime}(t)+q(t) y(t)\right]=\ell, 0 \leq \ell<\infty$. Since $q(t)$ is bounded, we have $\lim _{t \rightarrow \infty} r(t) y^{\prime \prime}(t)=\ell$. If $\ell>0$, then $y^{\prime}(t)>0$ for large $t$. This contradiction proves 
that $\ell=0$. Consequently, $\lim _{t \rightarrow \infty} y^{\prime \prime}(t)=0$. This completes the proof of the theorem.

Corollary 2.7. Let $\mathbf{( H )}$ and (2.10) hold, $\int_{\sigma}^{\infty}\left(\frac{g(t)}{t}\right)^{2} c(t) d t=\infty$. If for any $\mu \in(0,1 / 2), \mu\left(\frac{g(t)}{t}\right)^{2} c(t)-a(t) b(t)-b^{\prime}(t) \geq 0, a^{\prime}(t) \leq 0$, (2.13) is nonoscillatory, then every nonoscillatory solution of (1.1) along with its first and second derivatives tends to zero as $t \rightarrow \infty$.

This follows from Theorems 2.1 and 2.6.

The following result due to Potter ([13], Theorem 2.6) is needed.

Theorem 2.8. Suppose that $r$ and $q \in C^{1}((\sigma, \infty), R), r$ is positive and $q$ is nonnegative in $(\sigma, \infty)$ and

$$
\int_{\sigma_{1}}^{\infty} \frac{1}{r(t)} d t=\infty, \sigma_{1}>\sigma
$$

If $L=\lim _{t \rightarrow \infty}\left\{[r(t) q(t)]^{-1 / 2}\right\}$ exists and $L>2$, then equation (2.13) is nonoscillatory, where $r$ and $q$ are defined in (2.2).

Example. Consider

$$
\begin{aligned}
y^{\prime \prime \prime}(t)+\frac{1}{t^{2}} y^{\prime \prime}(t)+\frac{1}{t-1} & \left(\frac{2}{t^{2}}-\frac{1}{t^{3}}\right) y^{\prime}(t) \\
& +\frac{1}{e}\left(1-\frac{1}{t^{2}}+\frac{(2 t-1)}{(t-1) t^{3}}\right) y(t-1)=0
\end{aligned}
$$

for $t \geq 2$. In this case $L>2$ and hence, by Theorem 2.8, the second order differential equation

$$
z^{\prime \prime}(t)+\frac{1}{t^{2}} z^{\prime}(t)+\frac{1}{t-1}\left(\frac{2}{t^{2}}-\frac{1}{t^{3}}\right) z(t)=0
$$

is nonoscillatory. It is easy to check that all conditions of Corollary 2.7 are satisfied and hence all nonoscillatory solutions of (2.20) along with their first and second derivatives tend to zero as $t \rightarrow \infty$. In particular, $y(t)=e^{-t}$ is a nonoscillatory solution of $(2.20)$.

Remark 3. Our Theorem 2.6 improves Theorem 2.6 in [11].

Remark 4. Erbe [2] obtained several results for the bounded solutions only. Our Theorem 2.6 is an improvement of Theorem 2.2 due to Erbe [2].

\section{ACKNOWLEDGEMENT}

The author is thankful to the referee for his valuable remarks and suggestions in preparing the final version of the manuscript. 


\section{REFERENCES}

1. J. Džurina, Asymptotic properties of the third order delay differential equations. Nonlinear Anal. 26(1996), No. 1, 33-39.

2. L. ERBE, Oscillation and asymptotic behavior of solutions of third order differential delay equations. SIAM J. Math. Anal. 7(1976), No. 4, 491-500.

3. M. Gregus and M. Gera, Some results in the theory of a third-order linear differential equation. Ann. Polon. Math. 42 (1983), 93-102.

4. M. Gregus, J. R. Graef, and M. Gera, Oscillating nonlinear third order differential equations. Nonlinear Anal. 28(1997), No. 10, 1611-1622.

5. M. S. KeEnER, On the solutions of certain linear nonhomogeneous second-order differential equations. Applicable Anal. 1(1971), No. 1, 57-63.

6. T. Kusano and M. Naito, Comparison theorems for functional-differential equations with deviating arguments. J. Math. Soc. Japan 33(1981), No. 3, 509-532.

7. A. C. Lazer, The behavior of solutions of the differential equation $y^{\prime \prime \prime}+p(x) y^{\prime}+q(x) y=$ 0. Pacific J. Math. 17(1966), 435-466.

8. N. PARHI and S. PADHI, On asymptotic behavior of delay-differential equations of third order. Nonlinear Anal. 34(1998), No. 3, 391-403.

9. N. PARHI and S. PADHI, Asymptotic behaviour of a class of third order delay-differential equations. Math. Slovaca 50(2000), No. 3, 315-333.

10. N. PARHI and S. PADHI, On oscillatory linear third order differential equations. J. Indian Math. Soc. (N.S.) 69(2002), No. 1-4, 113-128.

11. N. PARHI and S. PADHI, Asymptotic behaviour of solutions of third order delaydifferential equations. Indian J. Pure Appl. Math. 33(2002), No. 10, 1609-1620.

12. Y. P. Singh, The asymptotic behavior of solutions of linear third order differential equations. Proc. Amer. Math. Soc. 20(1969), 309-314.

13. C. A. Swanson, Comparison and oscillation theory of linear differential equations. Mathematics in Science and Engineering, Vol. 48. Academic Press, New York-London, 1968.

14. W. F. Trench, Canonical forms and principal systems for general disconjugate equations. Trans. Amer. Math. Soc. 189(1973), 319-327.

(Received 1.07.2003; revised 26.07.2004)

Author's address:

Department of Applied Mathematics

Birla Institute of Technology

Mesra, Ranchi 835215

India

E-mail: ses_2312@yahoo.co.in

Current address:

Department of Mathematics and Statistics

Mississippi State University

Mississippi State, MS 39762

U.S.A 